\title{
Enhanced Photocatalytic Degradation of Phenol Using Urchin-Like ZnO Microrod-Reduced Graphene Oxide Composite under Visible-Light Irradiation
}

\author{
S. Mary Margaret, Albin John P. Paul Winston, S. Muthupandi, P. Shobha, \\ and P. Sagayaraj
}

Loyola College (Autonomous), Nungambakkam, Chennai, Tamil Nadu, India

Correspondence should be addressed to P. Sagayaraj; sagayaraj1962@gmail.com

Received 2 February 2021; Revised 8 June 2021; Accepted 26 June 2021; Published 26 July 2021

Academic Editor: Baskaran Rangasamy

Copyright (c) 2021 S. Mary Margaret et al. This is an open access article distributed under the Creative Commons Attribution License, which permits unrestricted use, distribution, and reproduction in any medium, provided the original work is properly cited.

\begin{abstract}
In this study, visible-light-driven $\mathrm{ZnO}$ microrod-rGO heterojunction composites were successfully synthesized via a facile and scalable hydrothermal process. The prepared photocatalyst heterojunction was examined using different techniques including $\mathrm{XRD}$, SEM, FTIR, UV-Vis spectroscopy, and TGA to reveal their crystal phase, morphology, and other optical properties. The photocatalytic performance of the obtained $\mathrm{ZnO}-\mathrm{rGO}$ composites was measured by the photodegradation of phenol under visible light illumination. The addition of graphene over the catalyst exhibited an enhanced photocatalytic activity for phenol degradation due to its high surface area and decreasing rate of electron-hole separation. Kinetic studies proved that the degradation of phenol process happened by following the pseudo-first-order kinetic model. The effective conditions for degradation of phenol using $\mathrm{ZnO}-\mathrm{rGO}$ composite were $0.2 \mathrm{~g} \mathrm{~L}^{-1}$ catalyst dose, $\mathrm{pH}-4$, and initial concentration $20 \mathrm{ppm}$ of phenol solution. Comparing with $\mathrm{ZnO}$ microrods, the heterojunction composite degraded the organic pollutants of phenol solution up to $84.2 \%$ of efficiency displaying the highest photocatalytic activity, whereas urchin-like $\mathrm{ZnO}$ catalyst exhibited much less photocatalytic activity for phenol degradation under visible light irradiation. This result envisages immense properties, showing a great potential industrial application for the removal of phenolic wastewater.
\end{abstract}

\section{Introduction}

In today's industrialization, environmental pollution has been booming day by day. Industries that use plenty of water include textile companies, refining petroleum, automotive manufacturing, and primary metals [1]. The discharge from these industries produces plenty of organic contamination which makes severe damages to the ecosystem especially to the aqueous system by decreasing the concentration of oxygen dissolved in the environment. Among the biorecalcitrant toxic compounds, phenol is the most harmful organic compound which is a ubiquitous water pollutant that plays a vital role in industries such as pharmaceuticals, coal conversion, paint, cresols, dyeing, and pulpmill [2]. The European Union regulation has declared the maximum permitted amount of phenolic compounds in freshwater as $0.5 \mathrm{mg} \mathrm{L}^{-1}$ [3].
Therefore, there has been a growing interest to eliminate these contaminations from the industrial water and make it usable for the ecological systems like plant, animal, and human beings. Thus, researchers have done a lot of work to develop fascinating methods for the removal of water pollutants in this area including the approaches like ion floatation, sonocatalytic degradation, photocatalytic degradation, swirling jet-induced method, hydrodynamic cavitation, electrochemical oxidation, and advanced oxidation processes [4-11]. Among these, heterogeneous photodegradation is the most remarkable and effective method for converting toxic organic pollutants into carbonaceous products [12].

Nowadays, many researchers prefer heterogeneous photodegradation process owing to its good electron conductivity, huge specific surface area, material consumption, high stability, high absorption, conduction empty band (CB), and 
electronic structure packed valance band (VB) [13-16]. The method of phenol degradation through semiconducting metal oxide has been under usage as photocatalyst materials involving $\mathrm{TiO}_{2}$ [17], $\mathrm{ZnO}$ [18], $\mathrm{V}_{2} \mathrm{O}_{5}$ [19], $\mathrm{Fe}_{3} \mathrm{O}_{4}$ [20], and $\mathrm{MgO}[21]$ and is well known and ascribed to the arrangement of electron-hole pair at the valance and conduction band after absorption of less amount of energy from UV or visible light.

Among semiconducting nano/microstructure, $\mathrm{ZnO}$ is a promising candidate with suitable bandgap energy of $\sim 3.4 \mathrm{eV}$ and versatile material for photodegradation under the removal of various types of organic dyes in an aqueous or gaseous medium [22]. It has been confirmed that $\mathrm{ZnO}$ can exhibit high photocatalytic degradation than $\mathrm{TiO}_{2}$ for some dyes owing to its economic viability and ecofriendly nature [23]. These are diverse experimental methods for the synthesis of ZnO-rGO heterojunction materials [24], including solvothermal, hydrothermal, microwave synthesis, electrochemical deposition, precipitation method, and solution combustion synthesis. In this paper, a hydrothermal approach was used for synthesizing urchin-like $\mathrm{ZnO}$ with the addition of graphene material with the idea to enhance the photocatalytic performance. Furthermore, the morphology plays a significant role for the better performance of photocatalytic activity. In the recent studies, hierarchically ordered $\mathrm{ZnO}$ microstructures are found to exhibit high photocatalytic performance due to their high stability against aggregation and large specific surface area [25, 26]. Literature reviews suggest that graphene sheet enhances various properties such as large values of Young modulus, extremely high surface area, high visible light optical transparency, superior mobility of charge carriers $\left(200,000 \mathrm{~cm}^{2} \mathrm{~V}^{-1} \mathrm{~s}^{-1}\right)$, and thermal conductivity $[27,28]$. Thus, reduced graphene oxide(rGO-) based metal oxide photocatalytic materials have recently gained a lot of attention among the scientists to improve the charge transfer at the interface; it reduces the recombination of charge carriers on the surface of catalysts and offers high performance to absorb pollutants. These multifunctional heterojunctions have been demonstrated to be outstanding candidate fruits in fields like photocatalytic degradation of organic pollutants over visible light irradiation. Research has been conducted for several decades on $\mathrm{ZnO}$ based nanocomposite due to its better photocatalytic performance and high surface area, but the complexity to recover from the reaction system and agglomeration are two major problems that restrict its practical application $[29,30]$. Thus, microsized $\mathrm{ZnO}$ with a high surface area is an alternative pathway to overcome these problems.

For instance, Qin et al. reported $\mathrm{ZnO}$ microsphere-rGO nanocomposites for photodegradation of methylene blue pigment under UV irradiation [31]. Pant et al. synthesized $\mathrm{ZnO}$ microflowers arranged on reduced graphene oxide of MB dye over UV irradiation [32]. To the best of our knowledge, there are limited works which used urchin-like $\mathrm{ZnO}$ microrod-rGO composites as a heterojunction for degradation of phenol in visible light irradiation. Herein, we report the synthesis of urchin-like $\mathrm{ZnO}$ microrod-rGO composites by a facile and environmentally friendly hydrothermal method. The synthesized composite was examined and char- acterized for photocatalytic degradation of phenol under visible light illumination. Moreover, the effect of special parameters including optical, magnetic, photoconductivity, phenol concentration, catalyst dosage, and photocatalytic properties of urchin-like $\mathrm{ZnO}$ microrod-rGO composite was also investigated.

\section{Materials and Methods}

2.1. Materials. Graphite, zinc nitrate hexahydrate $\left(\mathrm{Zn}\left(\mathrm{NO}_{3}\right)_{2} \cdot 6 \mathrm{H}_{2} \mathrm{O}\right)$, polyethylene glycol (PEG 4000), ammonium hydroxide $\left(\mathrm{NH}_{3} \cdot \mathrm{H}_{2} \mathrm{O}\right)$, potassium persulfate $\left(\mathrm{K}_{2} \mathrm{~S}_{2} \mathrm{O}_{8}\right)$, sulfuric acid $\left(\mathrm{H}_{2} \mathrm{SO}_{4}\right)$, phosphorus pentoxide $\left(\mathrm{P}_{2} \mathrm{O}_{5}\right)$, hydrochloric acid $(\mathrm{HCl})$, potassium permanganate $\left(\mathrm{KMnO}_{4}\right)$, ethylene glycol $\left(\mathrm{C}_{2} \mathrm{H}_{6} \mathrm{O}_{2}\right)$, hydrogen peroxide $\left(\mathrm{H}_{2} \mathrm{O}_{2}\right)$, and ascorbic acid $\left(\mathrm{C}_{6} \mathrm{H}_{8} \mathrm{O}_{6}\right)$ were purchased from Aldrich and used as received.

2.2. Preparation of Graphene Oxide (GO). Graphene oxide was synthesized from graphite powder by using the modified Hummers method [33]. Typically, $3 \mathrm{~g}$ of graphite, $2 \mathrm{~g}$ of $\mathrm{P}_{2} \mathrm{O}_{5}$, and $2 \mathrm{~g}$ of $\mathrm{K}_{2} \mathrm{~S}_{2} \mathrm{O}_{8}$ were added into $24 \mathrm{~mL}$ of concentrated $\mathrm{H}_{2} \mathrm{SO}_{4}$ under stirring at $95^{\circ} \mathrm{C}$ for $6 \mathrm{~h}$. The mixture was cooled down to normal room temperature and $1 \mathrm{~L}$ of deionized (DI) water was added into the mixture and kept under ageing for $48 \mathrm{~h}$. The mixture was then washed, filtered, and dried out to obtain the black powder. Subsequently, $15 \mathrm{~g}$ of $\mathrm{KMnO}_{4}$ and $125 \mathrm{~mL}$ of concentrated $\mathrm{H}_{2} \mathrm{SO}_{4}$ were added into the mixture and kept in an ice bath below $10^{\circ} \mathrm{C}$ and magnetically stirred at $40^{\circ} \mathrm{C}$ for $3 \mathrm{~h}$. The obtained grey sample was diluted with the dropwise addition of $10 \mathrm{~mL}$ of $\mathrm{H}_{2} \mathrm{O}_{2}$ followed by the slow addition of $0.5 \mathrm{~L}$ of DI water to quench the solution, and the colour of the solution became yellowish. The obtained product was centrifuged, cleaned with $\mathrm{HCl}$ to remove impurities, and washed with DI water. The final product was dried at $40^{\circ} \mathrm{C}$ in a vacuum oven to attain the GO sample.

2.3. Preparation of Reduced Graphene Oxide ( $r G O)$. Reduced graphene oxide ( $\mathrm{rGO}$ ) was synthesized from graphite oxide (GO) by using ascorbic acid as a reductant [34]. $0.1 \mathrm{mg} / \mathrm{mL}$ of GO solution and $0.1 \mathrm{M}$ ascorbic acid were mixed and kept at $70^{\circ} \mathrm{C}$ on a heating shield and stirred for $35 \mathrm{~min}$; the colour of the solution turned from yellow to black. The product was centrifuged and dried at $120^{\circ} \mathrm{C}$ in a vacuum.

2.4. Preparation of the Urchin-Like $\mathrm{ZnO}$ Microrod-rGO Composite Materials. In a typical experiment, $10.8 \mathrm{~g}$ $\mathrm{Zn}\left(\mathrm{NO}_{3}\right)_{2} \cdot 6 \mathrm{H}_{2} \mathrm{O}, 2.5$ wt. \% of rGO, $30 \mathrm{~mL}$ of $\mathrm{C}_{2} \mathrm{H}_{6} \mathrm{O}_{2}$, and $0.6 \mathrm{~g}$ PEG 4000 were dissolved in distilled water $(120 \mathrm{~mL})$. Then, $\mathrm{NH}_{3} \cdot \mathrm{H}_{2} \mathrm{O}$ was added dropwise until the $\mathrm{pH}$ adjusted to 8 . After being magnetically stirred for $35 \mathrm{~min}$, the solution was transferred into a $250 \mathrm{~mL}$ Teflon-lined stainless steel autoclave. The autoclave was kept at $120^{\circ} \mathrm{C}$ for $24 \mathrm{~h}$ in an oven and then cooled down to the room temperature naturally. The product was washed with distilled water and absolute ethanol several times and dried at $60^{\circ} \mathrm{C}$ for $24 \mathrm{~h}$.

Finally, the product of urchin-like $\mathrm{ZnO}$ microrod-rGO was obtained. 
2.5. Evaluation of Photocatalytic Activity. The degradation reaction of urchin-like $\mathrm{ZnO}$ microrod-rGO of the prepared catalysts was conducted by measuring the photodegradation of the phenol in aqueous solution $(10 \mathrm{mg} / \mathrm{L})$ under visible light illumination at ambient temperature. The adsorption process was kept under a dark region as a batch process. Typically, $30 \mathrm{mg}$ of photocatalyst was added to $50 \mathrm{~mL}$ of the aqueous solution and stirred for some time to evade residue of the catalyst. Prior to irradiation, the experimental set-up was placed in a complete dark portion and magnetically stirred for $60 \mathrm{~min}$ in the dark to achieve adsorption-desorption equilibrium. Subsequently, the photocatalytic reaction was in progress by the exposure of a $50 \mathrm{~W}$ tungsten lamp as a visible light source. The distance between source and photoreaction sample was $20 \mathrm{~cm}$, and $5 \mathrm{~mL}$ of sample was taken out with the help of a syringe $(\sim 5 \mathrm{~mL})$ at time interval of each $15 \mathrm{~min}$. The mixture solutions were then centrifuged, and the supernatant of the dye was analysed by using a UV-Vis spectrophotometer (Shimadzu 2450 series, Japan). The degradation efficiency of the phenol can be defined as follows:

$$
D(\%)=\left(\frac{1-C_{t}}{C_{0}}\right) \times 100 \%,
$$

where $C_{0}$ is the initial dye concentration $(\mathrm{mg} / \mathrm{L})$ at time $t=0$ and $C_{t}$ is the residual concentration of phenol $(\mathrm{mg} / \mathrm{L})$ at different irradiation intervals $t$, respectively. The photodegradation follows pseudo-first-order kinetics, which can be expressed as follows:

$$
-\ln \left(\frac{C_{t}}{C_{0}}\right)=k_{\mathrm{app}} t
$$

where $k\left(\mathrm{~min}^{-1}\right)$ is the rate constant of photodegradation [35].

2.6. Characterization. The X-ray diffractogram patterns of the prepared samples were recorded using an X-ray diffraction system (XRD $3003 \mathrm{TT}$ ) from 10 to $70^{\circ}$ with $\mathrm{Cu} \mathrm{K} \alpha$ $(1.5406 \AA)$ radiation. High-resolution scanning electron microscopy (HRSEM) was employed for morphological study by using GMS 5900, JEOL, Japan. Fourier transform infrared (FT-IR) spectrum studies were recorded using a PerkinElmer Spectrum 2 spectrometer in the wavenumber ranging from 4000 to $400 \mathrm{~cm}^{-1}$. Thermogravimetric (TGA) analysis was carried with the instrument (NETZSCH STA 449F3, Germany), to investigate the stability of samples at higher temperatures. A UV-Vis spectrometer (PerkinElmer Lambda 25) was used to collect the UV-visible absorption spectra of the dye samples.

\section{Results and Discussion}

3.1. X-Ray Diffraction Analysis. The recorded powder XRD patterns of the as-prepared $\mathrm{rGO}, \mathrm{ZnO}$, and the $\mathrm{ZnO}-\mathrm{rGO}$ composite is shown in Figure 1. The diffraction pattern of rGO shows a broad peak at $2 \theta$ value $25.6^{\circ}$ corresponding to the (002) planes of interlayer distance of $3.47 \AA$ [36]. The $\mathrm{XRD}$ peak of $\mathrm{ZnO}$ shows sharp and intense diffraction peaks at $2 \theta$ of $31.5^{\circ}, 34.1^{\circ}, 36^{\circ}, 47.2^{\circ}, 56.3^{\circ}, 62.6^{\circ}, 66.1^{\circ}, 67^{\circ}$, and $68.8^{\circ}$

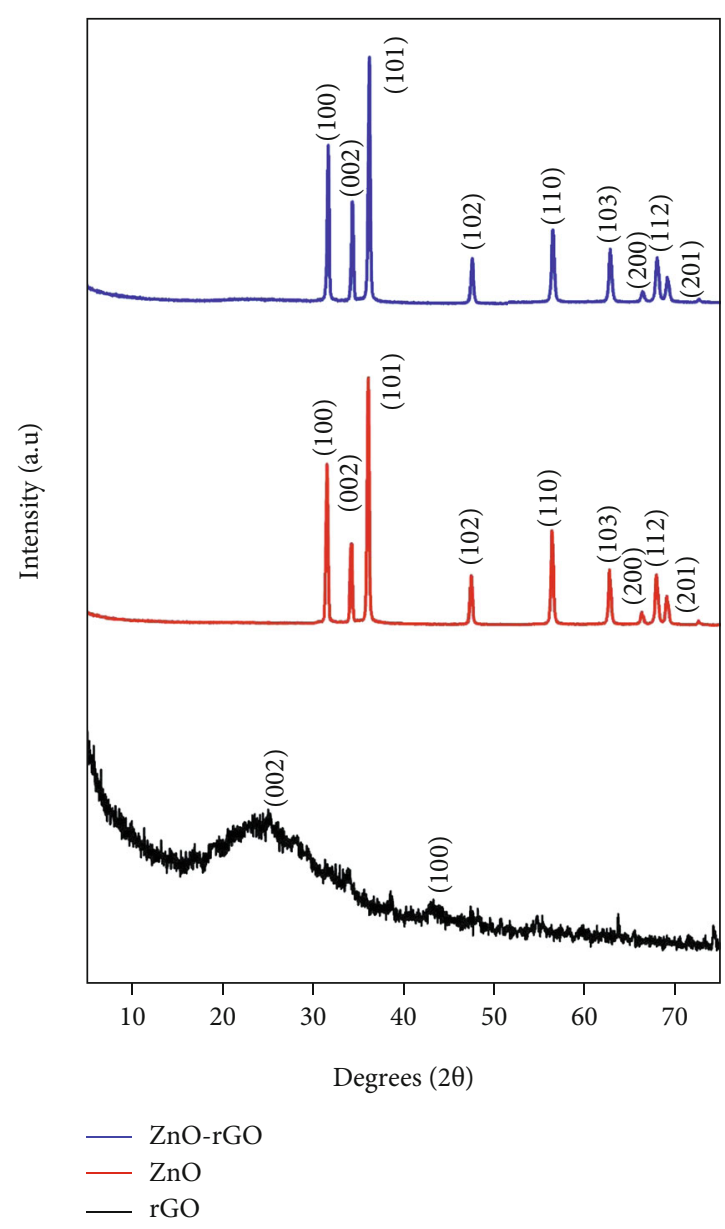

Figure 1: X-ray patterns of the rGO, $\mathrm{ZnO}$, and $\mathrm{ZnO}-\mathrm{rGO}$ composites.

and its corresponding lattice planes (100), (002), (101), (102), (110), (103), (002), (112), and (201), respectively. The asprepared urchin-like $\mathrm{ZnO}$ microstructure exhibited good crystalline nature with hexagonal wurtzite structure and is in agreement with the standard JCPDS card No: 36-1451 [37]. In addition, the $\mathrm{ZnO}-\mathrm{rGO}$ composite reveals a similar XRD pattern of $\mathrm{ZnO}$-related diffraction peaks with the rGO-related wide peak that confirms the heterojunction structure. However, the rGO peak is not seen in the $\mathrm{ZnO}$ rGO composite due to the relatively less intensity [38]. No proof of any other impurities is detected conforming that the $\mathrm{ZnO}-\mathrm{rGO}$ composite is successfully prepared.

3.2. Morphological Analysis. The morphologies and microstructures of $\mathrm{rGO}$, urchin-like $\mathrm{ZnO}$, and $\mathrm{ZnO}$ microrodrGO composites were investigated via HR-SEM and shown in Figures 2(a)-2(d), respectively. The compact wrinkledlike rGO sheet confirms the formation of the $2 \mathrm{D}$ structure which clearly indicates that the graphene oxide has been well reduced during the hydrothermal process (Figure 2(a)) [35]. The pure urchin-like $\mathrm{ZnO}$ (Figures 2(b) and 2(c)) with a diameter of about $10 \mu \mathrm{m}$ is self-assembled by micro/nanorods. It has a length of approximately 3-5 $\mu \mathrm{m}$ and a diameter of $600-800 \mathrm{~nm}$ as shown in Figure 2(c). The network of 


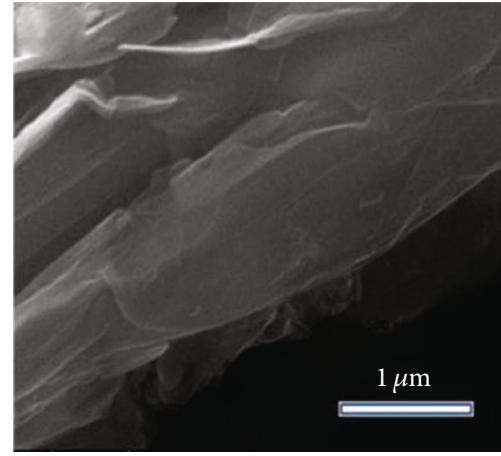

(a)

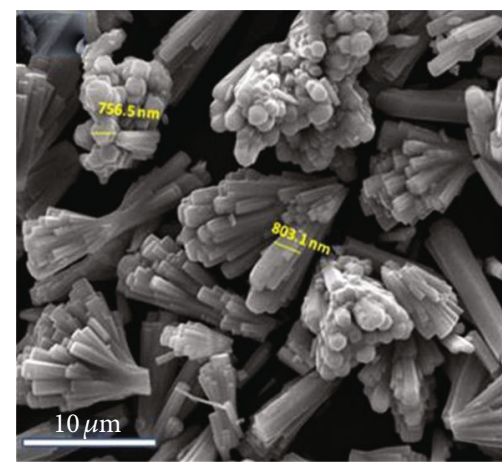

(c)

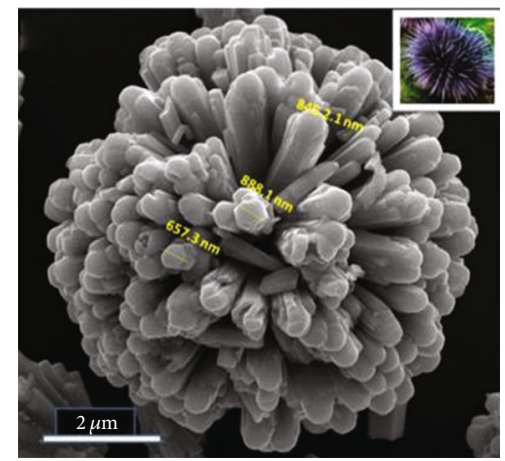

(b)

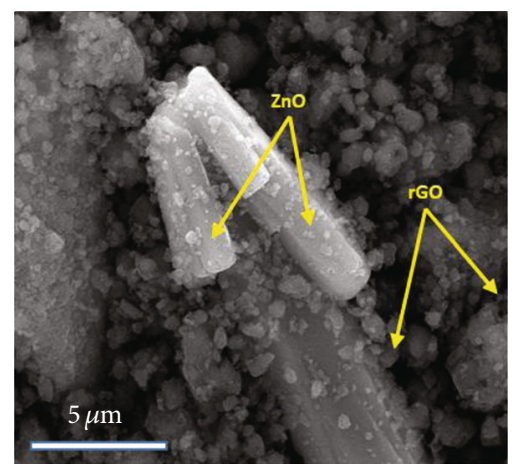

(d)

FIGURE 2: SEM images of (a) rGO, (b, c) urchin-like $\mathrm{ZnO}$ microrods, and (d) ZnO microrod-rGO composite.

urchin-like $\mathrm{ZnO}$ also shows a compact distribution of nanorods, and hence, it caused the well-ordered shape of nanorods. In the SEM image of ZnO-rGO (Figure 2(d)), it can be identified that urchin-like $\mathrm{ZnO}$ micro/nanorods are anchored and well dispersed on the rGO sheet. After the growth of $\mathrm{ZnO}$ micro/nanostructures on the $\mathrm{rGO}$, the graphene has changed its morphology due to larger size of $\mathrm{ZnO}$ particles. Hence, the larger size of composites will enhance a conductive to recycling and the more channels in the nanorods will provide better transportation of electrons during the process of photodegradation [39].

3.3. Fourier Transform Infrared (FT-IR) Analysis. Figure 3 shows the FT-IR spectra of rGO, urchin-like $\mathrm{ZnO}$, and ZnO-rGO composite. All samples exhibit a strong and wide absorption peak around $3200-3500 \mathrm{~cm}^{-1}$ which is attributed to the stretching of $\mathrm{O}-\mathrm{H}$ vibration of the amide group [40]. In the case of rGO, we observed that the absorption bands decreased distinctly to less intensity and oxygen functionalities disappeared, which conforms the surface of GO has been reduced to rGO [41]. Besides, the strong peaks at $459 \mathrm{~cm}^{-1}$ are assigned to the stretching vibration of the $\mathrm{Zn}-\mathrm{O}$ hexagonal phase [42]. The absorption peaks at $1627 \mathrm{~cm}^{-1}, 1385 \mathrm{~cm}^{-1}$, and $893 \mathrm{~cm}^{-1}$ correspond to the aromatic $\mathrm{C}=\mathrm{C}$ bond and skeletal ring vibrations from the graphitic domain, $\mathrm{C}-\mathrm{OH}$ stretching vibrations, and bending vibration of $\mathrm{C}-\mathrm{OH}$, respectively [40, 43]. But after hydrothermal reaction, the intensity of the peak significantly decreased. However, this peak was red shifted to $534 \mathrm{~cm}^{-1}$ in the $\mathrm{ZnO}-\mathrm{rGO}$ composite due to interactions between the urchin-like $\mathrm{ZnO}$ microstruc-

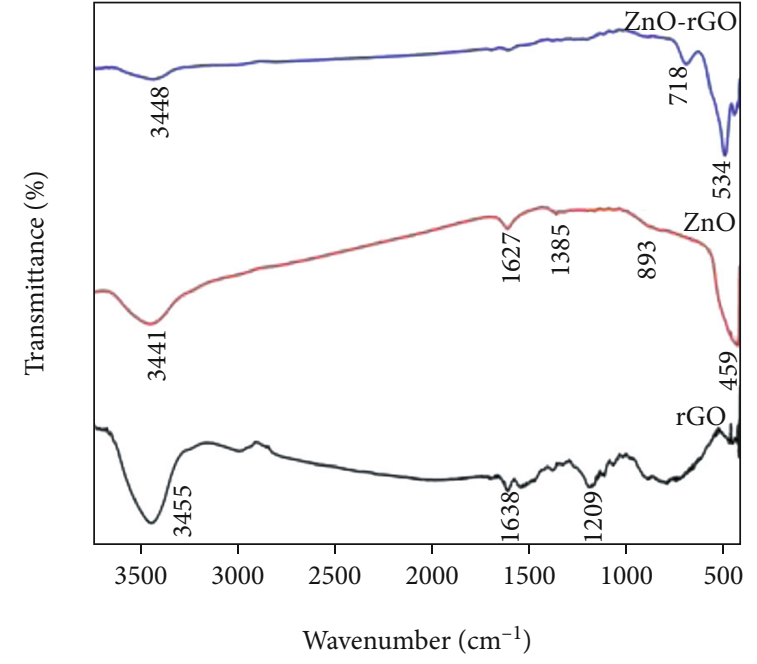

Figure 3: FT-IR spectra of the rGO, $\mathrm{ZnO}$, and $\mathrm{ZnO}-\mathrm{rGO}$ composites.

ture and residual epoxy and hydroxyl functional groups of the $\mathrm{rGO}[40,44,45]$.

3.4. UV-Visible Absorbance Analysis. To examine the optical properties of the as-synthesized materials, UV-Vis optical absorption spectra were recorded (Figure 4(a)). It is an effective way to study about surface defects and is strongly associated with the photocatalytic activity of $\mathrm{ZnO}$-rGO composites [46]. The strong characteristic absorbance peak at about $377 \mathrm{~nm}$ indicated highly crystalline and inherent bandgap 


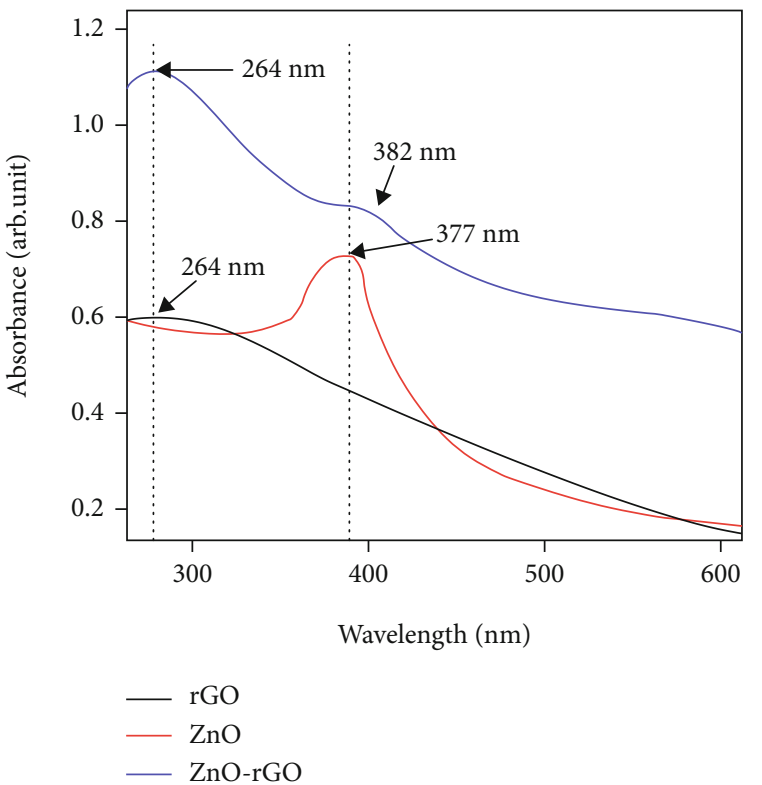

(a)

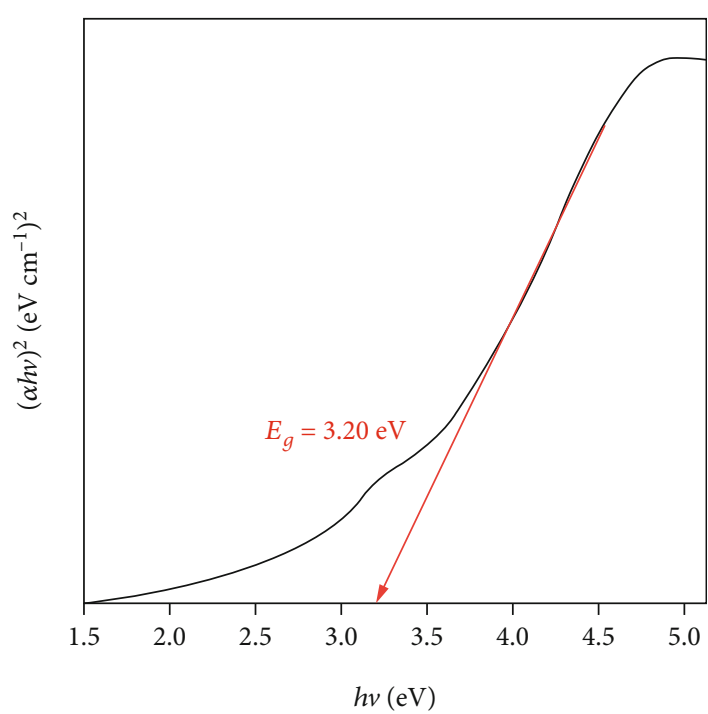

$h v(\mathrm{eV})$

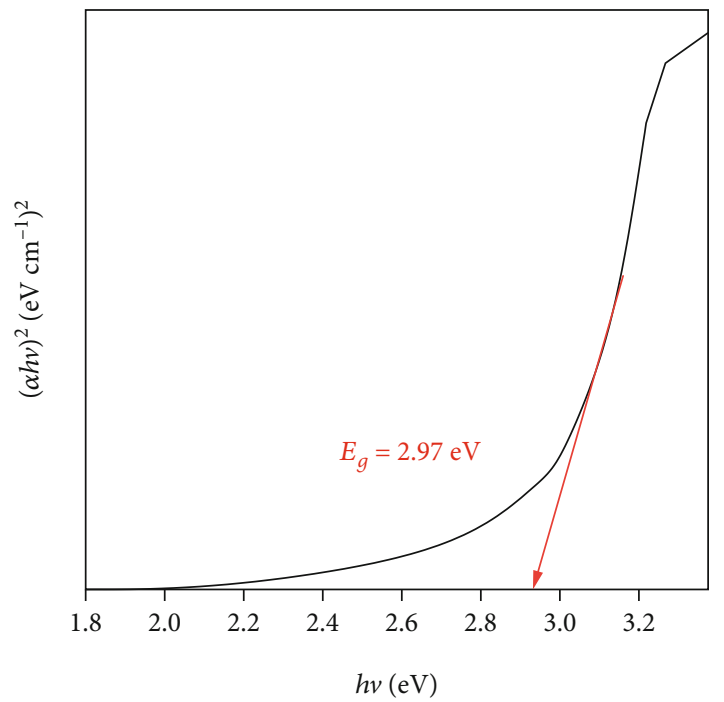

(c)

Figure 4: (a) UV-visible absorbance spectra for the as-synthesized rGO, ZnO, and ZnO-rGO; (b, c) Tauc plot to examine the bandgap of ZnO and $\mathrm{ZnO}-\mathrm{rGO}$.

absorption of $\mathrm{ZnO}$ particles [28]. rGO shows the absorption peak seen at $264 \mathrm{~nm}$ and is attributed to $\pi-\pi *$ orbital transition [47], while the characteristic spectra of ZnO-rGO composites show a peak at $264 \mathrm{~nm}$ which belongs to the rGO, and another absorption peak at $382 \mathrm{~nm}$ is attributed to $\mathrm{ZnO}$ microstructure. Therefore, the $\mathrm{ZnO}$ in the composite material got red shifted from 377 to $382 \mathrm{~nm}$ mainly due to the addition of rGO through $\mathrm{ZnO}$. The overall peak shows that the composite material of urchin-like $\mathrm{ZnO}$-rGO microstructure has higher absorption capacity than pure $\mathrm{ZnO}$ which is confirmed from the UV-spectrum. Thus, the higher absorption capacity plays an important role in the photocatalytic performance of visible light irradiation for dyes.
To estimate the bandgap energy of synthesized catalysts, the UV-visible absorbance spectra of samples were analysed by performing by the Kubelka-Munk theory. The material was calculated by plotting $(\alpha h v)^{2}$ versus $(h v)$ photon energy by using the following equation:

$$
\alpha h v=A(h v-E g)^{1 / 2},
$$

where $\alpha$ is the absorption coefficient of the catalyst, $v$ is the frequency of light, $h v$ is the photon energy, $A$ is the constant energy, and $E_{g}$ is the bandgap energy, respectively. From Figures $4(\mathrm{~b})$ and $4(\mathrm{c})$, the estimated bandgap energy values 


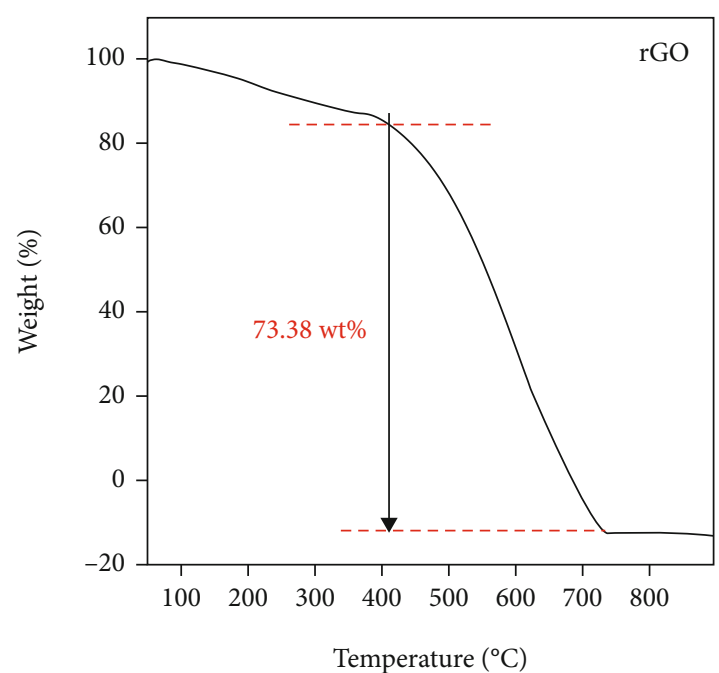

(a)

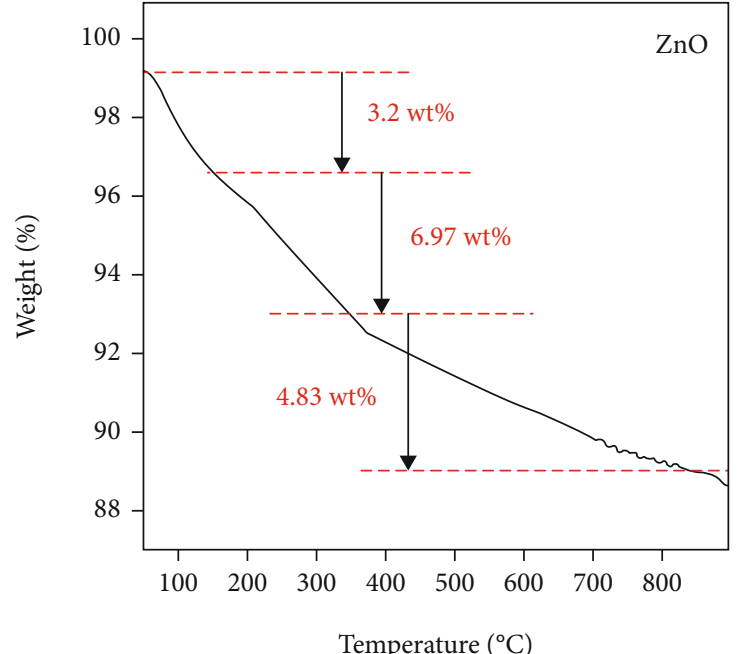

(b)

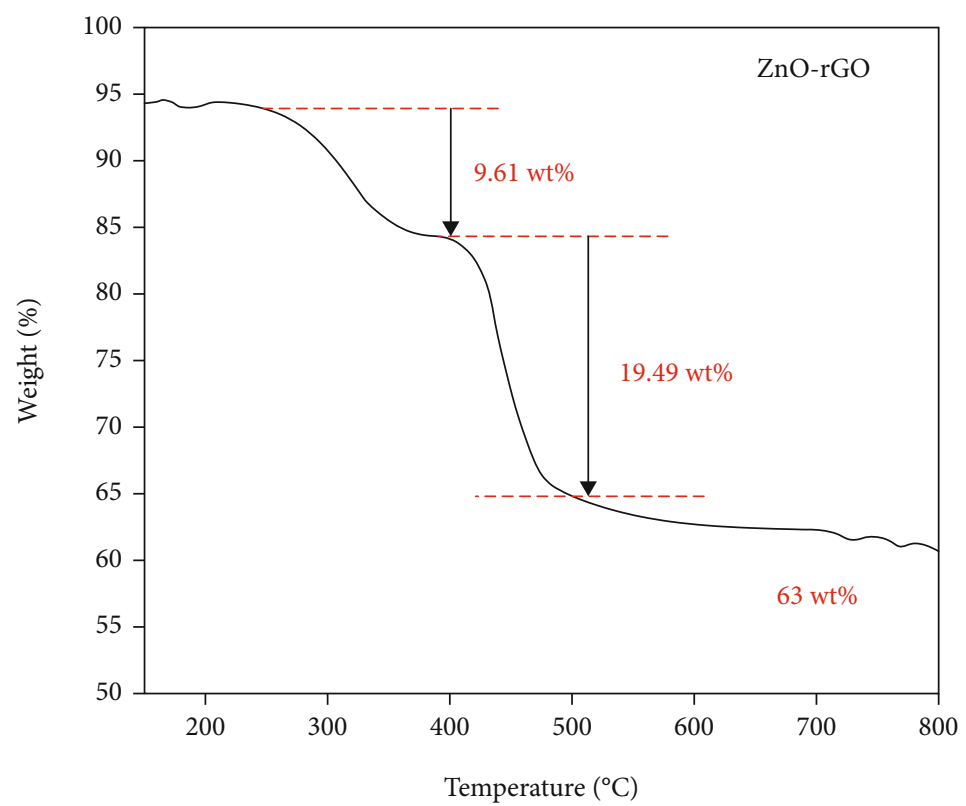

(c)

FIgUre 5: TGA curves of (a) rGO, (b) urchin-like $\mathrm{ZnO}$, and (c) ZnO-rGO composites.

for $\mathrm{ZnO}$ and $\mathrm{ZnO}-\mathrm{rGO}$ composites are $3.20 \mathrm{eV}$ and $2.97 \mathrm{eV}$, respectively. It can be seen that the $E_{g}$ value of the composite material is red shifted from the $E_{g}$ value of $\mathrm{ZnO}$ microstructure, suggesting the bandgap reduction of $\mathrm{ZnO}-\mathrm{rGO}$ catalyst, which can enhance high visible light absorption for the photocatalytic performance of phenol.

3.5. Thermogravimetric Analysis. As presented in Figure 5(a), the thermogram of rGO, the weight loss at about $73.38 \mathrm{wt}$. $\%$ mainly occurs at the range of $400^{\circ} \mathrm{C}$ and drops drastically due to the increasing temperature range from $410^{\circ} \mathrm{C}$ and $735^{\circ} \mathrm{C}$, indicating that rGO has a homogeneous composition distribution to the oxidation of carbon in air simultaneously. The residual weight of rGO which is about 12.66 wt. \% denotes that rGO is totally combusted [47].
From Figure 5(b), the TGA traces of $\mathrm{ZnO}$ one could observe the three weight loss in the range of $50-150^{\circ} \mathrm{C}$, $150-350^{\circ} \mathrm{C}$, and $350-600^{\circ} \mathrm{C}$. Weight loss of about $3.2 \mathrm{wt} \%$ from 50 to $150^{\circ} \mathrm{C}$ is related to the dehydration of surfaceadsorbed water molecules and removal of chemically absorbed alcohol. The weight loss of about $6.97 \mathrm{wt}$. \% from 150 to $350^{\circ} \mathrm{C}$ is due to the degradation of the organic molecules and the hydroxide groups. Therefore, annealing at above $350^{\circ} \mathrm{C}$ assured the formation of $\mathrm{ZnO}$ particles [48]. The TGA trace of ZnO-rGO composites is illustrated in Figure 5(c). The weight loss of about $9.61 \mathrm{wt}$. \% above $250^{\circ} \mathrm{C}$ is ascribed to be oxidation of graphene under atmosphere. A significant mass loss of about $19.49 \mathrm{wt}$. \% is observed at the temperature range above $400^{\circ} \mathrm{C}-510^{\circ} \mathrm{C}$ which is supposedly due to decomposition of $\mathrm{rGO}$ in the composite [49]. Based on the above result, we can conclude 


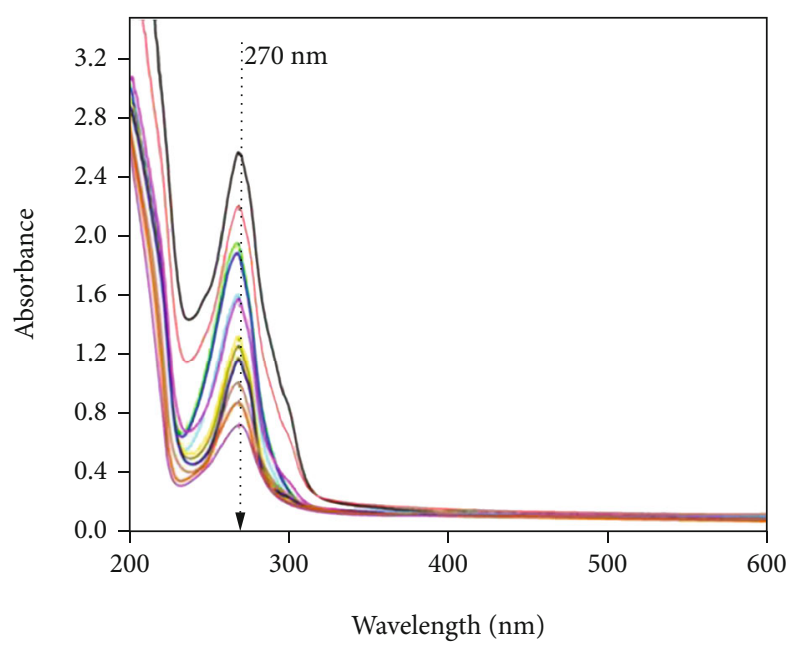

(a)

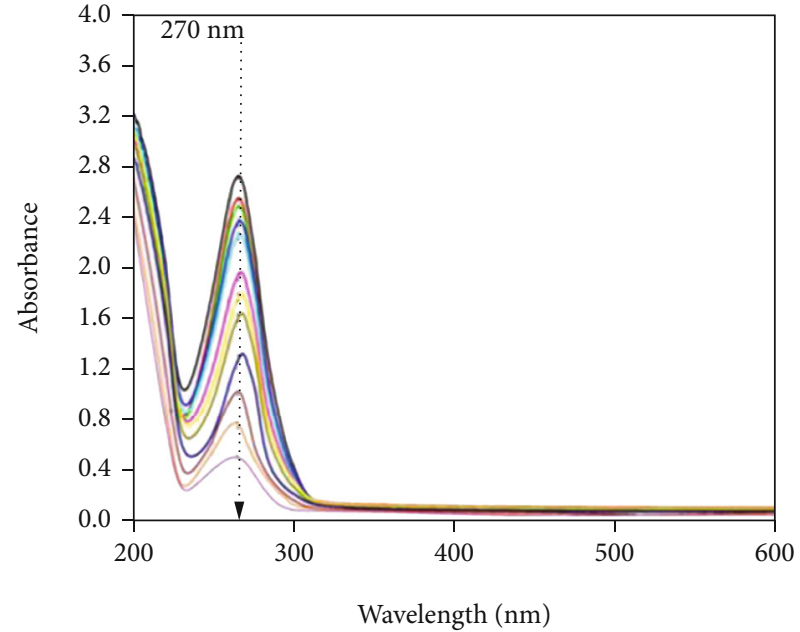

Phenol
- $0 \mathrm{~min}$
$15 \mathrm{~min}$
$-30 \mathrm{~min}$
$45 \mathrm{~min}$
$60 \mathrm{~min}$

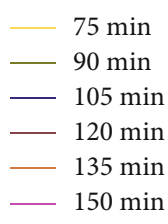

b)

Figure 6: (a, b) UV-Vis absorption spectrum changes of phenol at every 15 min time interval of (a) urchin-like $\mathrm{ZnO}$ and (b) ZnO-rGO composite, respectively.

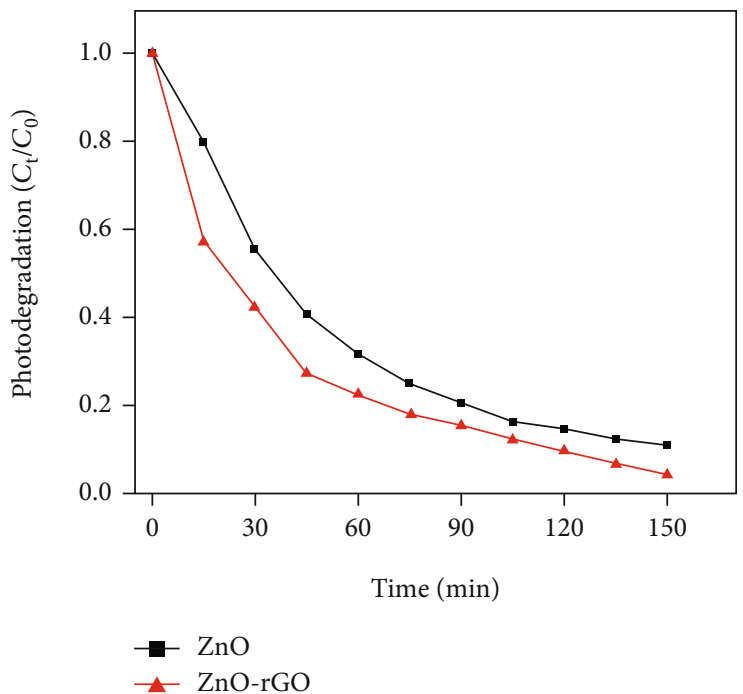

(a)

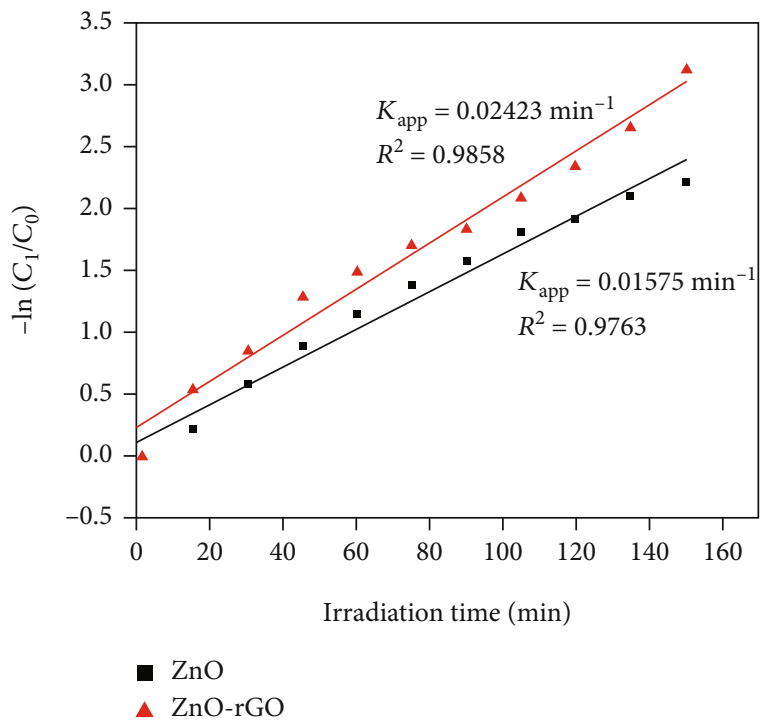

(b)

FIgURE 7: (a) Study of linear apparent first-order kinetics for the photodegradation of phenol solution using urchin-like ZnO and ZnO microrod-rGO catalyst under visible light illumination. (b) Photodegraded efficiencies for the ZnO-rGO nano/microcomposites and urchin-like $\mathrm{ZnO}$, variation in $-\ln \left(C_{t} / C_{0}\right)$ as function of irradiation time and linear fits of photocatalysts. The kinetic rate of phenol degradation by the photocatalysts was also investigated.

that the residual weight ratio of $\mathrm{ZnO}-\mathrm{rGO}$ composites is about 63 wt. $\%$ at $520^{\circ} \mathrm{C}$, respectively.

3.6. Photocatalytic Activity. The photocatalytic performance of urchin-like $\mathrm{ZnO}$ and $\mathrm{ZnO}$ microrod-rGO composites was examined for their photodegradation of phenol solution as a model pollutant under visible light irradiation. Figure 6 represents the time-dependent UV-Vis absorption spectrum of the aqueous solution of phenol which leads to the decrease in its absorbance reduction with various duration. Prior to the irradiation, the sample was stirred magnetically in the completely dark area for $60 \mathrm{~min}$ to attain the adsorption equilibrium of phenol solution over the photocatalyst. The photodegradation was carried out for $150 \mathrm{~min}$, and the 


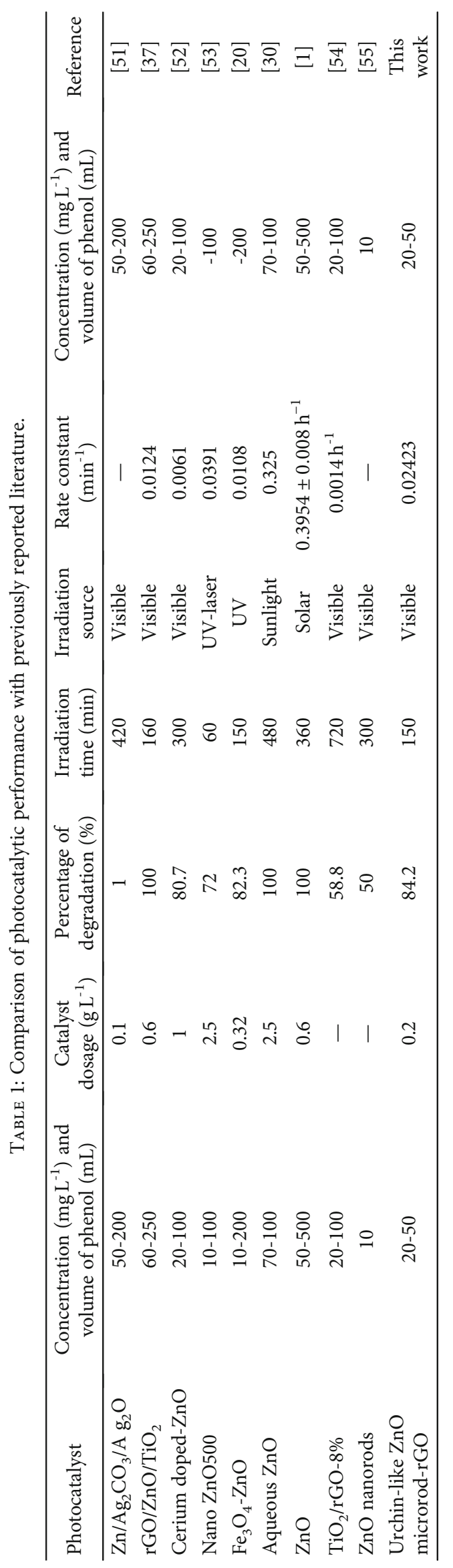


degradation rate of phenol solution has shown a maximum absorption wavelength at $\lambda_{\max }=270 \mathrm{~nm}$ [3].

In general, the concentration of phenol or absorbance gradually decreases with the increasing illumination time in the presence of urchin-like $\mathrm{ZnO}$ and $\mathrm{ZnO}-\mathrm{rGO}$ photocatalyst, respectively. After irradiation, the concentration of phenol is reduced, implying the decolourization of photocatalyst, and ascribed to the oxidation of phenol in the existence of $\mathrm{OH}$ radicals. The degradation result shows that the $\mathrm{ZnO}$ nano/micro-rGO heterojunction degrades the organic pollutants of phenol solution up to $\sim 84.2 \%$ and urchin-like $\mathrm{ZnO}$ catalyst degrades $\sim 71.5 \%$ in just 150 min under similar operating conditions. Therefore, the $\mathrm{ZnO}$ nano/microrodrGO composites exhibit better photocatalytic activity than those of urchin-like $\mathrm{ZnO}$ microrods. As shown in Figures 6(a) and 6(b), the typical absorption peak at $270 \mathrm{~nm}$ does not change its wavelength even after $150 \mathrm{~min}$, which indicated that the prepared photocatalyst of phenol is active under visible light irradiation.

According to literature, the addition of rGO in the catalysts favours a larger surface area to bind a phenol molecule through the $\pi-\pi$ conjugation with face-to-face direction [50]. The dispersion of $\mathrm{ZnO}-\mathrm{rGO}$ composites enhances the surface of oxygen species, improves the electron-hole pairs, and lowers the bandgap. Moreover, the absorption edge of $\mathrm{ZnO}-\mathrm{rGO}$ composites shifted to the broad wavelength after $135 \mathrm{~min}$ and provides a favourable absorption of phenol under visible light irradiation. The observed normalized temporal concentration changes $\left(C_{t} / C_{0}\right)$ of phenol during photodegradation activity of prepared photocatalysts (urchin-like $\mathrm{ZnO}$ and $\mathrm{ZnO}$ microrod-rGO composites) vs. time graph are shown in Figure $7(\mathrm{a})$. It is clear that $\mathrm{ZnO}-\mathrm{rGO}$ with $84.2 \%$ demonstrates an outstanding enhancement in the photodegradation of phenol compared with urchin-like $\mathrm{ZnO}$ microrods.

Pseudo-first-order reaction. The reaction was calculated from the slope of $-\ln \left(C_{t} / C_{0}\right)$ and reaction time, $t$ (Figure 7(b)). From this graph, we can understand that urchin-like $\mathrm{ZnO}$ and $\mathrm{ZnO}$ microrod-rGO composites exhibit linear fit lines and confirm that the photocatalytic degradation reaction follows pseudo-first-order reaction kinetics. As shown in Figure 7(b), the rate constant of urchin-like $\mathrm{ZnO}$ exhibits a $K_{\mathrm{app}}$ of $0.015 \mathrm{~min}^{-1}$, whereas the $\mathrm{ZnO}$ microrod-rGO composites reach into $0.024 \mathrm{~min}^{-1}$, which is about 1.5 times faster rate than that of pure $\mathrm{ZnO}$ microrods. The higher rate constant of $\mathrm{ZnO}$ microrod-rGO composites yields a large number of photoelectrons which enhance the photocatalysis. Herein, to better assess, the performance of the synthesized catalyst was compared with the detoxification of phenol reported in literature studies (Table 1). It is concluded that $\mathrm{ZnO}$ microrod-rGO composites are an efficient catalyst for decontamination of phenol over visible light irradiation. It may be beneficial for the use of industrial applications due to its cost-effective and great performance.

\section{Conclusion}

In this investigation, we successfully synthesized a costeffective and highly active $\mathrm{ZnO}$ microrod-rGO composite of heterojunction via a facile hydrothermal process. The $\mathrm{ZnO}$ rGO $(2.97 \mathrm{eV})$ displayed a narrow bandgap when compared to urchin-like $\mathrm{ZnO}(3.20 \mathrm{eV})$ and also showed strong visible light absorption and an enhanced photoresponse to visible light regions. The photodegradation efficiency of the composite reached $84.2 \%$, and the catalyst performance was evaluated by phenol degradation under the irradiation of visible light. The $\mathrm{ZnO}-\mathrm{rGO}$ composite incorporated with additional electronic channels with the presence of urchin-like structure can absorb a large quantity of phenol because of its large surface area and transfer of electrons faster due to the presence of rGO in the composite. Therefore, the obtained results suggest that the prepared $\mathrm{ZnO}-\mathrm{rGO}$ composite is a promising candidate for photodegradation process under visible-lightdriven photocatalyst for decontamination of toxic compounds and effluents.

\section{Data Availability}

The data supporting this work is available from the corresponding author upon request.

\section{Conflicts of Interest}

The authors declare that they have no conflicts of interest.

\section{Supplementary Materials}

The pictorial representation of this work has been described in the form of graphical abstract available in the supplementary information. (Supplementary Materials)

\section{References}

[1] N. Yusoff, S.-A. Ong, L.-N. Ho, Y.-S. Wong, and W. Khalik, "Degradation of phenol through solar-photocatalytic treatment by zinc oxide in aqueous solution," Desalination and Water Treatment, vol. 54, pp. 1621-1628, 2014.

[2] N. C. Saha, F. Bhunia, and A. Kaviraj, "Toxicity of phenol to fish and aquatic ecosystems," Bulletin of Environmental Contamination and Toxicology, vol. 63, no. 2, pp. 195-202, 1999.

[3] F. Hayati, A. A. Isari, M. Fattahi, B. Anvaripour, and S. Jorfi, "Photocatalytic decontamination of phenol and petrochemical wastewater through $\mathrm{ZnO} / \mathrm{TiO}_{2}$ decorated on reduced graphene oxide nanocomposite: influential operating factors, mechanism, and electrical energy consumption," RSC Advances, vol. 8, no. 70, pp. 40035-40053, 2018.

[4] A. Lakshmanan, P. Surendran, S. SakthyPriya et al., "Effect of fuel content on nonlinear optical and antibacterial activities of $\mathrm{Zn} / \mathrm{Cu} / \mathrm{Al}_{2} \mathrm{O}_{4}$ nanoparticles prepared by microwaveassisted combustion method," Journal of King Saud University - Science, vol. 32, no. 2, pp. 1382-1389, 2020.

[5] M. Ahmad, E. Ahmed, Z. L. Hong, W. Ahmed, A. Elhissi, and N. R. Khalid, "Photocatalytic, sonocatalytic and sonophotocatalytic degradation of rhodamine $\mathrm{B}$ using $\mathrm{ZnO} / \mathrm{CNT}$ s composites photocatalysts," Ultrasonics Sonochemistry, vol. 21, no. 2, pp. 761-773, 2014.

[6] S. Fang, K. Lv, Q. Li, H. Ye, D. Du, and M. Li, "Effect of acid on the photocatalytic degradation of rhodamine B over $\mathrm{g}-\mathrm{C}_{3} \mathrm{~N}_{4}$," Applied Surface Science, vol. 358, pp. 336-342, 2015. 
[7] X. Wang, J. Wang, P. Guo, W. Guo, and C. Wang, "Degradation of rhodamine $\mathrm{B}$ in aqueous solution by using swirling jet-induced cavitation combined with $\mathrm{H}_{2} \mathrm{O}_{2}$," Journal of Hazardous Materials, vol. 30, pp. 486-491, 2009.

[8] S. Ravichandran and G. Ramalingam, "Synthesis, optical and morphological studies of sol-gel derived $\mathrm{ZnO} / \mathrm{PVP}$ one dimensional nano-composite," Journal of Nanoscience and Nanotechnology, vol. 1, pp. 39-43, 2013.

[9] K. Mishra and P. Gogate, "Intensification of degradation of rhodamine B using hydrodynamic cavitation in the presence of additives," Separation and Purification Technology, vol. 75, no. 3, pp. 385-391, 2010.

[10] I. Oller, S. Malato, and J. A. Sánchez-Pérez, "Combination of advanced oxidation processes and biological treatments for wastewater decontamination-a review," Science of The Total Environment, vol. 409, no. 20, pp. 4141-4166, 2011.

[11] G. Theophil Anand, D. Renuka, R. Ramesh et al., "Green synthesis of $\mathrm{ZnO}$ nanoparticle using Prunus dulcis (almond gum) for antimicrobial and supercapacitor applications," Surfaces and Interfaces, vol. 17, p. 100376, 2019.

[12] M. Antonopoulou, E. Evgenidou, D. Lambropoulou, and I. Konstantinou, "A review on advanced oxidation processes for the removal of taste and odor compounds from aqueous media," Water Research, vol. 53, pp. 215-234, 2014.

[13] M. R. S. Joice, T. M. David, and P. Wilson, "WO3 nanorods supported on mesoporous $\mathrm{TiO}_{2}$ nanotubes as onedimensional nanocomposites for rapid degradation of methylene blue under visible light irradiation," Journal of Physical Chemistry C, vol. 123, no. 45, pp. 27448-27464, 2019.

[14] H. Huang, H. Ouyang, T. Han, H. Wang, and X. Zheng, "Construction of carbon quantum dots/single crystal $\mathrm{TiO}_{2}$ nanosheets with exposed $\{001\}$ and $\{101\}$ facets and their visible light driven catalytic activity," RSC Advances, vol. 9, no. 7, pp. 3532-3541, 2019.

[15] S. G. Kumar and L. G. Devi, "Review on modified $\mathrm{TiO}_{2}$ photocatalysis under UV/visible light: selected results and related mechanisms on interfacial charge carrier transfer dynamics," The Journal of Physical Chemistry. A, vol. 115, no. 46, pp. 13211-13241, 2011.

[16] G. Ramalingam, C. Ragupathi, K. Kaviyarasu et al., "Up-scalable synthesis of size-controlled white-green emitting behavior of core/shell (CdSe/ZnS) quantum dots for LED applications," Journal of Nanoscience and Nanotechnology, vol. 19, no. 7, pp. 4026-4032, 2019.

[17] A. L. Linsebigler, G. Lu, and J. T. Yates, "Photocatalysis on $\mathrm{TiO}_{2}$ surfaces: principles, mechanisms, and selected results," Chemical Reviews, vol. 95, no. 3, pp. 735-758, 1995.

[18] S. Anas, S. Rahul, K. B. Babitha, R. V. Mangalaraja, and S. Ananthakumar, "Microwave accelerated synthesis of zinc oxide nanoplates and their enhanced photocatalytic activity under UV and solar illuminations," Applied Surface Science, vol. 355, pp. 98-103, 2015.

[19] H. Yin, K. Yu, C. Song, R. Huang, and Z. Zhu, "Synthesis of Au-decorated V2O5@ZnO heteronanostructures and enhanced plasmonic photocatalytic activity," ACS Applied Materials \& Interfaces, vol. 6, no. 17, pp. 14851-14860, 2014.

[20] X. Feng, H. Guo, K. Patel, H. Zhou, and X. Lou, "High performance, recoverable $\mathrm{Fe}_{3} \mathrm{O}_{4} \mathrm{ZnO}$ nanoparticles for enhanced photocatalytic degradation of phenol," Chemical Engineering Journal, vol. 244, pp. 327-334, 2014.
[21] S. Jorfi, G. Barzegar, M. Ahmadi et al., "Enhanced coagulationphotocatalytic treatment of acid red 73 dye and real textile wastewater using UVA/synthesized MgO nanoparticles," Journal of Environmental Management, vol. 15, pp. 111-118, 2016.

[22] P. S. Chauhan, R. Kant, A. Rai, A. Gupta, and S. Bhattacharya, "Facile synthesis of $\mathrm{ZnO} / \mathrm{GO}$ nanoflowers over Si substrate for improved photocatalytic decolorization of MB dye and industrial wastewater under solar irradiation," Materials Science in Semiconductor Processing, vol. 89, pp. 6-17, 2019.

[23] A. Meng, J. Shao, X. Fan, J. Wang, and Z. Li, "Rapid synthesis of a flower-like $\mathrm{ZnO} / \mathrm{rGO} / \mathrm{Ag}$ micro/nano-composite with enhanced photocatalytic performance by a one-step microwave method," RSC Advances, vol. 4, no. 104, pp. 60300 60305, 2014.

[24] P. Van Tuan, T. T. Phuong, V. T. Tan, S. X. Nguyen, and T. N. Khiem, "In-situ hydrothermal fabrication and photocatalytic behavior of $\mathrm{ZnO} /$ reduced graphene oxide nanocomposites with varying graphene oxide concentrations," Materials Science in Semiconductor Processing, vol. 115, p. 105114, 2020.

[25] V. A. Tu and V. A. Tuan, "A facile and fast solution chemistry synthesis of porous $\mathrm{ZnO}$ nanoparticles for high efficiency photodegradation of tartrazine," Vietnam Journal of Chemistry, vol. 56, no. 2, pp. 214-219, 2018.

[26] T. H. Le, Q. D. Truong, T. Kimura et al., "Synthesis of hierarchical porous $\mathrm{ZnO}$ microspheres and its photocatalytic deNOx activity," Ceramics International, vol. 38, no. 6, pp. 5053-5059, 2012.

[27] M. Darvishi, F. Jamali-Paghaleh, M. Jamali-Paghaleh, and J. Seyed-Yazdi, "Facile synthesis of $\mathrm{ZnO} / \mathrm{rGO}$ hybrid by microwave irradiation method with improved photoactivity," Surfaces and Interfaces, vol. 9, pp. 167-172, 2017.

[28] K. Huang, Y. H. Li, S. Lin et al., "A facile route to reduced graphene oxide-zinc oxide nanorod composites with enhanced photocatalytic activity," Powder Technology, vol. 257, pp. 113-119, 2014.

[29] S. Xu, L. Fu, T. S. H. Pham, A. Yu, F. Han, and L. Chen, "Preparation of $\mathrm{ZnO}$ flower/reduced graphene oxide composite with enhanced photocatalytic performance under sunlight," Ceramics International, vol. 41, no. 3, pp. 4007-4013, 2015.

[30] S. K. Pardeshi and A. B. Patil, "A simple route for photocatalytic degradation of phenol in aqueous zinc oxide suspension using solar energy," Solar Energy, vol. 82, no. 8, pp. 700-705, 2008.

[31] J. Qin, X. Zhang, C. Yang, M. Cao, M. Ma, and R. Liu, “ZnO microspheres-reduced graphene oxide nanocomposite for photocatalytic degradation of methylene blue dye," Applied Surface Science, vol. 392, pp. 196-203, 2017.

[32] H. R. Pant, C. H. Park, P. Pokharel, L. D. Tijing, D. S. Lee, and C. S. Kim, "ZnO micro-flowers assembled on reduced graphene sheets with high photocatalytic activity for removal of pollutants," Powder Technology, vol. 235, pp. 853-858, 2013.

[33] N. Cao and Y. Zhang, "Study of reduced graphene oxide preparation by hummers' method and related characterization," Journal of Nanomaterials, vol. 2015, Article ID 168125, 5 pages, 2015.

[34] E. Andrijanto, S. Shoelarta, G. Subiyanto, and S. Rifki, "Facile synthesis of graphene from graphite using ascorbic acid as reducing agent," AIP Conference Proceedings, vol. 1725, p. 20003, 2016.

[35] Y. Feng, N. Feng, Y. Wei, and G. Zhang, "An in situ gelatinassisted hydrothermal synthesis of $\mathrm{ZnO}$-reduced graphene 
oxide composites with enhanced photocatalytic performance under ultraviolet and visible light," RSC Advances, vol. 4, no. 16, pp. 7933-7943, 2014.

[36] M. Pusty, A. K. Rana, Y. Kumar, V. Sathe, S. Sen, and P. Shirage, "Synthesis of partially reduced graphene oxide/silver nanocomposite and its inhibitive action on pathogenic fungi grown under ambient conditions," ChemistrySelect, vol. 1, no. 14, pp. 4235-4245, 2016.

[37] H. Moussa, E. Girot, K. Mozet, H. Alem, G. Medjahdi, and R. Schneider, " $\mathrm{ZnO}$ rods/reduced graphene oxide composites prepared _via_a solvothermal reaction for efficient sunlightdriven photocatalysis," Applied Catalysis B: Environmental, vol. 185, pp. 11-21, 2016.

[38] H.-W. Wang, Z.-A. Hu, Y.-Q. Chang et al., "Design and synthesis of $\mathrm{NiCo}_{2} \mathrm{O}_{4}$-reduced graphene oxide composites for high performance supercapacitors," Journal of Materials Chemistry, vol. 21, no. 28, pp. 10504-10511, 2011.

[39] Y. Zhou, D. Li, L. Yang et al., "Preparation of 3D urchin-like $\mathrm{RGO} / \mathrm{ZnO}$ and its photocatalytic activity," Journal of Materials Science: Materials in Electronics, vol. 28, no. 11, pp. 7935-7942, 2017.

[40] H. N. Tien, V. H. Luan, L. T. Hoa et al., "One-pot synthesis of a reduced graphene oxide-zinc oxide sphere composite and its use as a visible light photocatalyst," Chemical Engineering Journal, vol. 229, pp. 126-133, 2013.

[41] L. Zhang, L. Du, X. Cai et al., "Role of graphene in great enhancement of photocatalytic activity of $\mathrm{ZnO}$ nanoparticlegraphene hybrids," Physica E: Low-dimensional Systems and Nanostructures, vol. 47, pp. 279-284, 2013.

[42] D. Gnanasangeetha and D. Saralathambavani, "One pot synthesis of zinc oxide nanoparticles via chemical and green method," Research Journal of Material Sciences, vol. 1, pp. 1-8, 2013.

[43] K. Rokesh, A. Nithya, K. Jeganathan, and K. Jothivenkatachalam, "A facile solid state synthesis of cone-like $\mathrm{ZnO}$ microstructure an efficient solar-light driven photocatalyst for rhodamine B degradation," Materials Today: Proceedings, vol. 3, no. 10, pp. 41634172, 2016.

[44] Q.-P. Luo, X.-Y. Yu, B.-X. Lei, H.-Y. Chen, D.-B. Kuang, and C.-Y. Su, "Reduced graphene oxide-hierarchical $\mathrm{ZnO}$ hollow sphere composites with enhanced photocurrent and photocatalytic activity," Journal of Physical Chemistry C, vol. 116, no. 14, pp. 8111-8117, 2012.

[45] Q. Zhang, C. Tian, A. Wu et al., "A facile one-pot route for the controllable growth of small sized and well-dispersed $\mathrm{ZnO}$ particles on GO-derived graphene," Journal of Materials Chemistry, vol. 22, no. 23, pp. 11778-11784, 2012.

[46] H.-B. Kim, D.-W. Jeong, and D.-J. Jang, "Morphology-tunable synthesis of $\mathrm{ZnO}$ microstructures under microwave irradiation: formation mechanisms and photocatalytic activity," CrystEngComm, vol. 18, no. 6, pp. 898-906, 2016.

[47] P. Liu, Y. Huang, and X. Zhang, "Cubic $\mathrm{NiFe}_{2} \mathrm{O}_{4}$ particles on graphene-polyaniline and their enhanced microwave absorption properties," Composites Science and Technology, vol. 107, pp. 54-60, 2015.

[48] J. Wang, G. Wang, S. Miao, J. Li, and X. Bao, "Graphene-supported iron-based nanoparticles encapsulated in nitrogendoped carbon as a synergistic catalyst for hydrogen evolution and oxygen reduction reactions," Faraday Discussions, vol. 176, pp. 135-151, 2014.

[49] F. B. Dejene, A. G. Ali, H. C. Swart et al., "Optical properties of $\mathrm{ZnO}$ nanoparticles synthesized by varying the sodium hydrox- ide to zinc acetate molar ratios using a sol-gel process," Central European Journal of Physics, vol. 9, no. 5, pp. 1321-1326, 2011.

[50] X. Zhou, T. Shi, and H. Zhou, "Hydrothermal preparation of $\mathrm{ZnO}$-reduced graphene oxide hybrid with high performance in photocatalytic degradation," Applied Surface Science, vol. 258, no. 17, pp. 6204-6211, 2012.

[51] N. Rosman, W. N. W. Salleh, A. F. Ismail et al., "Photocatalytic degradation of phenol over visible light active $\mathrm{ZnO} / \mathrm{Ag}_{2-}$ $\mathrm{CO}_{3} / \mathrm{Ag}_{2} \mathrm{O}$ nanocomposites heterojunction," Journal of Photochemistry and Photobiology A: Chemistry, vol. 364, pp. 602612,2018

[52] J.-C. Sin, S.-M. Lam, K.-T. Lee, and A. R. Mohamed, "Preparation of cerium-doped $\mathrm{ZnO}$ hierarchical micro/nanospheres with enhanced photocatalytic performance for phenol degradation under visible light," Journal of Molecular Catalysis A: Chemical, vol. 409, pp. 1-10, 2015.

[53] K. Hayat, M. A. Gondal, M. M. Khaled, S. Ahmed, and A. M. Shemsi, "Nano ZnO synthesis by modified sol gel method and its application in heterogeneous photocatalytic removal of phenol from water," Applied Catalysis A: General, vol. 393, no. 1-2, pp. 122-129, 2011.

[54] G. Wang, W. Guo, D. Xu, D. Liu, and M. Qin, "Graphene oxide hybridised $\mathrm{TiO}_{2}$ for visible light photocatalytic degradation of phenol," Symmetry (Basel), vol. 12, no. 9, article ???, 2020.

[55] J. Al-Sabahi, T. Bora, M. Al-Abri, and J. Dutta, "Controlled defects of zinc oxide nanorods for efficient visible light photocatalytic degradation of phenol," Materials (Basel), vol. 9, no. 4 , p. $238,2016$. 